\title{
Superconductivity Emerging near Quantum Critical Point of Valence Transition
}

\author{
Shinji Watanabe*1, Masatoshi Imada ${ }^{1,2}$ and Kazumasa Miyake ${ }^{3}$ \\ Institute for Solid State Physics, University of Tokyo, 5-1-5 Kashiwanoha, Kashiwa, Chiba 277-8581, \\ Japan $^{1}$ \\ PRESTO, Japan Science and Technology Agency, 4-1-8 Honcho, Kawaguchi, Saitama 332-0012, \\ Japan $^{2}$ \\ Division of Materials Physics, Department of Materials Engineering Science, Graduate School of \\ Engineering Science, Osaka University, Toyonaka, Osaka 560-8531, Japan ${ }^{3}$
}

(Received April 11, 2018)

\begin{abstract}
The nature of the quantum valence transition is studied in the one-dimensional periodic Anderson model with Coulomb repulsion between $\mathrm{f}$ and conduction electrons by the density-matrix renormalization group method. It is found that the first-order valence transition emerges with the quantum critical point and the crossover from the Kondo to the mixed-valence states is strongly stabilized by quantum fluctuation and electron correlation. It is found that the superconducting correlation is developed in the Kondo regime near the sharp valence increase. The origin of the superconductivity is ascribed to the development of the coherent motion of electrons with enhanced valence fluctuation, which results in the enhancement of the charge velocity, but not of the charge compressibility. Statements on the valence transition in connection with Ce metal and Ce compounds are given.
\end{abstract}

KEYWORDS: $\mathrm{CeCu}_{2} \mathrm{Ge}_{2}$, valence transition, quantum critical point, valence fluctuation, superconductivity, DMRG, periodic Anderson model

In $\mathrm{CeCu}_{2} \mathrm{Ge}_{2},{ }^{1}$ the superconducting transition temperature $T_{\mathrm{SC}}$ has a maximum in the pressure regime far from the antiferromagnetic (AF) quantum critical point (QCP), where the coefficient $A$ in the resistivity $\rho=\rho_{0}+A T^{n}$ drops rapidly for $n \sim 2$. Since $A$ is proportional to the effective $\operatorname{mass}^{2}\left(m^{*} / m\right)^{2}$ which is related to the f-electron number as $m^{*} / m=(1-$ $\left.n_{\mathrm{f}} / 2\right) /\left(1-n_{\mathrm{f}}\right){ }^{3}$ this implies that $T_{\mathrm{SC}}$ has a maximum around the sharp valence change of Ce. Similar behavior has been observed in the isostructural compound $\mathrm{CeCu}_{2} \mathrm{Si}_{2}{ }^{4}$ and in $\mathrm{CeCu}_{2}\left(\mathrm{Si}_{1-\mathrm{x}} \mathrm{Ge}_{\mathrm{x}}\right)_{2} .{ }^{5}$ Recently, an NMR measurement has revealed that $T_{\mathrm{SC}}$ increases under hydrostatic pressure in CeIrIn 5 although AF spin fluctuation is suppressed, ${ }^{6}$ suggesting the different mechanism for the superconductivity from ordinary AF spin fluctuation.

As known as the $\gamma$ - $\alpha$ transition, ${ }^{7}$ Ce metal shows the first-order valence transition in its temperature-pressure phase diagram. The valence change of Ce is due to the $4 \mathrm{f}$ level located near the Fermi level, which can hybridize easily with the conduction band by applying

*E-mail address: swata@issp.u-tokyo.ac.jp 
pressure. At the critical point, the valence susceptibility diverges as diverging density fluctuation in the liquid-gas transition. When the critical temperature is suppressed by controlling the chemical substitution and applying pressure, and enters the Fermi-degeneracy regime, diverging valence fluctuation is considered to be coupled with the Fermi-surface instability. This multiple instability in the quantum-degeneracy regime seems to be a key mechanism for understanding the instabilities observed in the above Ce compounds. However, theoretical understanding of the mechanism has not been fully achieved although a pioneering work on the valence-fluctuation-mediated superconductivity ${ }^{8}$ and later discussions ${ }^{9}$ have been made.

In this Letter, we clarify the nature of the first-order valence transition as well as the electronic states near the quantum critical point. We show that the superconducting correlation becomes developed in the Kondo regime near the sharp valence crossover on the basis of the density-matrix renormalization group $(\mathrm{DMRG})^{10}$ calculation for the one-dimensional periodic Anderson model

$$
\begin{aligned}
H & =-t \sum_{i \sigma}\left(c_{i \sigma}^{\dagger} c_{i+1 \sigma}+c_{i+1 \sigma}^{\dagger} c_{i \sigma}\right)+\varepsilon_{\mathrm{f}} \sum_{i \sigma} n_{i \sigma}^{\mathrm{f}} \\
& +V \sum_{i \sigma}\left(f_{i \sigma}^{\dagger} c_{i \sigma}+c_{i \sigma}^{\dagger} f_{i \sigma}\right)+U \sum_{i=1}^{N} n_{i \uparrow}^{\mathrm{f}} n_{i \downarrow}^{\mathrm{f}} \\
& +U_{\mathrm{fc}} \sum_{i=1}^{N} n_{i}^{\mathrm{f}} n_{i}^{\mathrm{c}} .
\end{aligned}
$$

Here, the notation is standard and the number operator is defined by $n_{i \sigma}^{\mathrm{a}}=a_{i \sigma}^{\dagger} a_{i \sigma}$ and $n_{i}^{\mathrm{a}}=n_{i \uparrow}^{\mathrm{a}}+n_{i \downarrow}^{\mathrm{a}}$ for $\mathrm{a}=\mathrm{f}$ and $\mathrm{c}$. The last term is the Coulomb repulsion between $\mathrm{f}$ and conduction electrons, which is important in causing the valence transition. ${ }^{8,11,12}$

The filling $n$ is defined by $n=\left(n_{\mathrm{f}}+n_{\mathrm{c}}\right) / 2$ with $n_{\mathrm{a}}=\sum_{i=1}^{N}\left\langle n_{i}^{\mathrm{a}}\right\rangle / N$ for a $=\mathrm{f}$ and c with $N$ being the number of lattice sites. We note that half filling is realized when $n=1$ in this definition. In this Letter, we consider the case $n=7 / 8, t=1, V=0.1$ and $U=100$. In the DMRG calculation, we have kept the number of states up to 1500 and the system sizes up to $N=80$ with the open-boundary condition.

Figure 1(a) shows the f-electron-number $n_{\mathrm{f}}$ vs the f-level $\varepsilon_{\mathrm{f}}$ for $U_{\mathrm{fc}}=7.0$, which has been obtained by a linear extrapolation to $N \rightarrow \infty$ using the data for $N=24,32,40,48,56,64,72$ and 80 . We see that a jump in $n_{\mathrm{f}}$ appears at $\varepsilon_{\mathrm{f}}=-5.6395$, which indicates the first-order quantum phase transition, since a jump in $n_{\mathrm{f}}$ results in the level crossing of the ground states by the relation $n_{\mathrm{f}}=\partial\langle H\rangle / \partial \varepsilon_{\mathrm{f}}$. The first-order valence transition (FOVT) between the Kondo state with $n_{\mathrm{f}} \simeq 1$ and the mixed valence $(\mathrm{MV})$ state with $n_{\mathrm{f}} \simeq 1 / 4(=2(1-n)$ for $1 / 2$ $\leq n \leq 1)$ is caused by $U_{\mathrm{fc}}$, since a large $U_{\mathrm{fc}}$ forces the electrons to pour into either the f level or the conduction band.

When $U_{\mathrm{fc}}$ decreases, the magnitude of the jump in $n_{\mathrm{f}}$ decreases. We see that the jump 

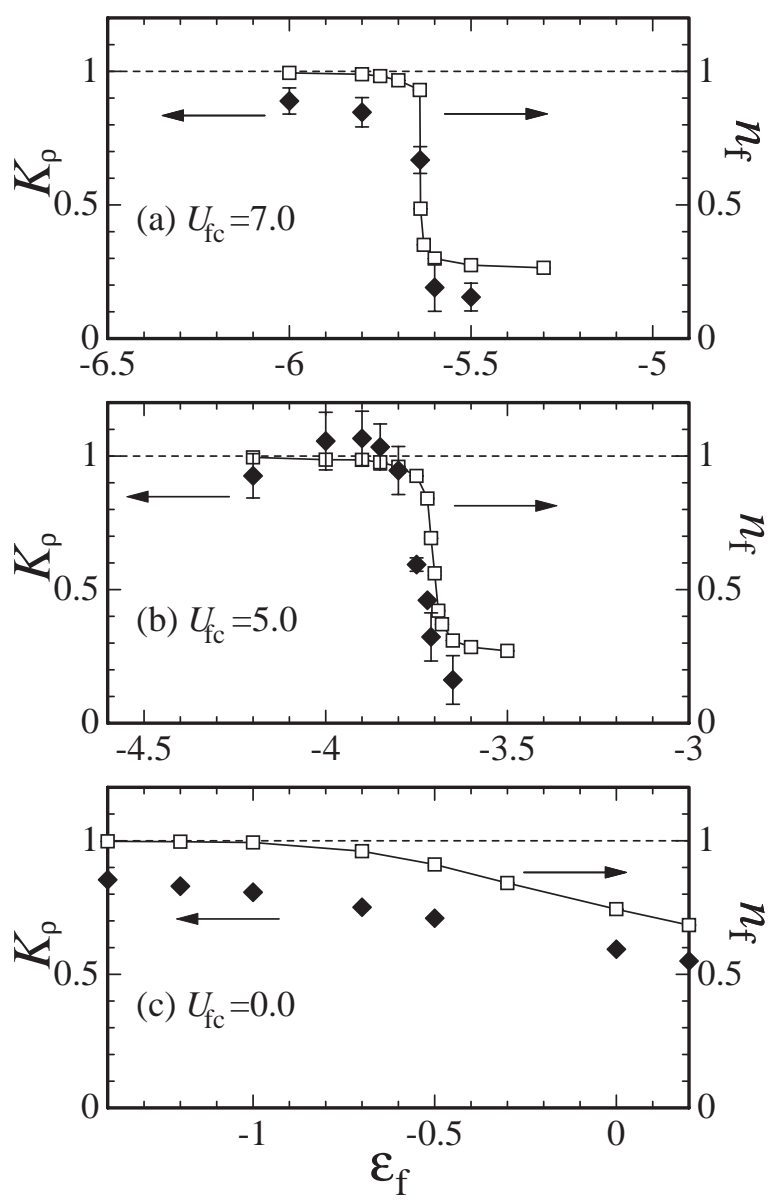

Fig. 1. $n_{\mathrm{f}}$ vs $\varepsilon_{\mathrm{f}}$ (open square) and $K_{\rho}$ vs $\varepsilon_{\mathrm{f}}$ (solid diamond) for $t=1, V=0.1$ and $U=100$ at $n=7 / 8$ for (a) $U_{\mathrm{fc}}=7.0$, (b) 5.0 and (c) 0.0 .

is invisible at $U_{\mathrm{fc}}=5.0$ and the sharp crossover from the Kondo to MV states appears in Fig. 1(b). These results imply that the QCP at which both the jump disappears and the valence susceptibility $\chi_{\mathrm{f}} \equiv-\partial^{2}\langle H\rangle / \partial \varepsilon_{\mathrm{f}}^{2}=-\partial n_{\mathrm{f}} / \partial \varepsilon_{\mathrm{f}}$ diverges, exists between $U_{\mathrm{fc}}=5.0$ and 7.0. As $U_{\mathrm{fc}}$ is set to be smaller than 5.0, the change in $n_{\mathrm{f}}$ becomes smoother and finally a gradual crossover appears at $U_{\mathrm{fc}}=0$ as seen in Fig. 1(c).

To determine the location of the QCP, we have calculated the jump of the f-electron number, $\Delta n_{\mathrm{f}} \equiv n_{\mathrm{f}}^{\mathrm{K}}-n_{\mathrm{f}}^{\mathrm{MV}}$, at the FOVT point as a function of $\varepsilon_{\mathrm{f}}$ for several $U_{\mathrm{fc}}$ values. Here, the superscripts $\mathrm{K}$ and MV specify the Kondo and mixed-valence states, respectively. Then, the QCP is estimated at $\left(\varepsilon_{\mathrm{f}}^{\mathrm{QCP}}, U_{\mathrm{fc}}^{\mathrm{QCP}}\right) \simeq(-4.5,5.9)$ at which the jump disappears. In the slave-boson mean-field $(\mathrm{MF})$ theory ${ }^{14}$ the $\mathrm{QCP}$ is identified at $\left(\varepsilon_{\mathrm{f} M F}^{\mathrm{QCP}}, U_{\mathrm{fc} M F}^{\mathrm{QCP}}\right)=(0.19,0.98)$. The critical nature of the valence transition will be discussed in detail in a separate paper. ${ }^{14}$

We summarize the ground-state phase diagram in the $U_{\mathrm{fc}}-\varepsilon_{\mathrm{f}}$ plane in Fig. 2. The solid line with solid diamonds represents the FOVT line determined by the DMRG, and the dashed line with open squares represents the point at which $\chi_{\mathrm{f}}$ has a maximum, which indicates the 
crossover with enhanced valence fluctuation. Here, we note that the slope of the FOVT line is expressed as the ratio of the jump in $n_{\mathrm{f}}$ and $\left\langle n_{i}^{\mathrm{f}} n_{i}^{\mathrm{c}}\right\rangle$ at the transition by the 'ClaudiusClapeyron relation', ${ }^{13} \delta U_{\mathrm{fc}} / \delta \varepsilon_{\mathrm{f}}=-\left(n_{\mathrm{f}}^{\mathrm{K}}-n_{\mathrm{f}}^{\mathrm{MV}}\right) /\left(C_{\mathrm{fc}}^{\mathrm{K}}-C_{\mathrm{fc}}^{\mathrm{MV}}\right)$, where $C_{\mathrm{fc}}$ is defined as $C_{\mathrm{fc}} \equiv$ $\sum_{i=1}^{N}\left\langle n_{i}^{\mathrm{f}} n_{i}^{\mathrm{c}}\right\rangle / N$. For $U_{\mathrm{fc}} \gg U_{\mathrm{fc}}^{\mathrm{QCP}}, \delta U_{\mathrm{fc}} / \delta \varepsilon_{\mathrm{f}}$ is shown to be $-1 / n_{\mathrm{c}}^{\mathrm{K}}$ in the MF theory, ${ }^{14}$ which coincides with the equation of the energy valance of the one-body f-electron energy at the FOVT; $\varepsilon_{\mathrm{f}}+U_{\mathrm{fc}} n_{\mathrm{c}}^{\mathrm{K}} \sim \mu$, with $\mu$ being the chemical potential. ${ }^{8}$ However, it should be stressed that the correct slope for $U_{\mathrm{fc}} \gg U_{\mathrm{fc}}^{\mathrm{QCP}}$ is given as $\delta U_{\mathrm{fc}} / \delta \varepsilon_{\mathrm{f}}=-1$ by inserting the atomic-limit values of $n_{\mathrm{f}}^{\mathrm{K}}=1, n_{\mathrm{f}}^{\mathrm{MV}}=1 / 4, C_{\mathrm{fc}}^{\mathrm{K}}=3 / 4$ and $C_{\mathrm{fc}}^{\mathrm{MV}}=0$ into the thermodynamic relation. This discrepancy is due to the insufficiency of the description of the MV state by the MF theory.

Here, we give the following rigorous remarks on the valence transition: For general filling of $1 / 2 \leq n \leq 1$, the maximum value of the jump of $n_{\mathrm{f}}$, which is realized for large values of $\left|\varepsilon_{\mathrm{f}}\right|$ and $U_{\mathrm{fc}}$, is given by $\left.\Delta n_{\mathrm{f}}\right|_{\max }=2 n-1$. This is in sharp contrast to the MF result, that always predicts the $4 \mathrm{f}^{1}\left(\mathrm{Ce}^{3+}\right)$ to $4 \mathrm{f}^{0}\left(\mathrm{Ce}^{4+}\right)$ transition; i.e., $\left.\Delta n_{\mathrm{f}}\right|_{\max }=1$. The slope of the FOVT line for large values of $\left|\varepsilon_{\mathrm{f}}\right|$ and $U_{\mathrm{fc}}$ is expressed by $\delta U_{\mathrm{fc}} / \delta \varepsilon_{\mathrm{f}}=-1$, irrespective of total filling $n .{ }^{14}$ It is noted that these results hold even in the square- and cubic-lattice systems.

We also point out that the reason why the FOVT is difficult to realize in the Ce compounds can be understood by the smallness of $U_{\mathrm{fc}}$ : In Ce metal, $U_{\mathrm{fc}}$ is large because of the on-site $4 \mathrm{f}-5 \mathrm{~d}$ interaction, whereas in Ce compounds $U_{\mathrm{fc}}$ is considered to be smaller even if the coordination number between Ce and surrounding atoms is taken into account, since $U_{\mathrm{fc}}$ corresponds to the intersite $4 \mathrm{f}-(\mathrm{d}$, or $\mathrm{p})$ interaction.

We have confirmed that at both sides of the FOVT, the peak structure appears at $q=\pi / 4$ in the Fourier component of $\left\langle S_{i}^{\mathrm{a} z}\right\rangle$ for $\mathrm{a}=\mathrm{f}$ and $\mathrm{c}$ when we apply a small magnetic field as a perturbation at the edge sites. The above period of the Friedel oscillations corresponds to $2 k_{\mathrm{F}}^{\text {large }}=\pi / 4$, since $k_{\mathrm{F}}^{\text {large }}=(1+3 / 4) \pi / 2=7 \pi / 8$. The large Fermi surfaces detected in the Kondo and MV states are consistent with the existence of the QCP, since by detouring around the QCP, the Kondo and MV states can be adiabatically connected, with Luttinger's sum rule satisfied.

In Fig. 2, the solid line with solid triangles represents the FOVT line determined by the slave boson MF theory. It is evident that the QCP (solid circle) identified by the DMRG is shifted from the QCP (open diamond) by the MF to the large- $\left|\varepsilon_{\mathrm{f}}\right|$ and large- $U_{\mathrm{fc}}$ direction. We also find no evidence of the phase separation at the FOVT, such as negative compressibility, and an inhomogeneous charge distribution in the DMRG results for the ground state. This result is due to the fact that the order parameter $n_{\mathrm{f}}$ is not a conserving quantity, which is in sharp contrast to the MF theory where the FOVT is accompanied by the phase separation. ${ }^{14}$ These results indicate that the phase separation is destabilized by quantum fluctuation and electron correlation, and as a result the crossover regime from the Kondo to MV states is strongly stabilized. In connection to the experiments of the Ce compounds such as $\mathrm{CeCu}_{2} \mathrm{Ge}_{2}$, 


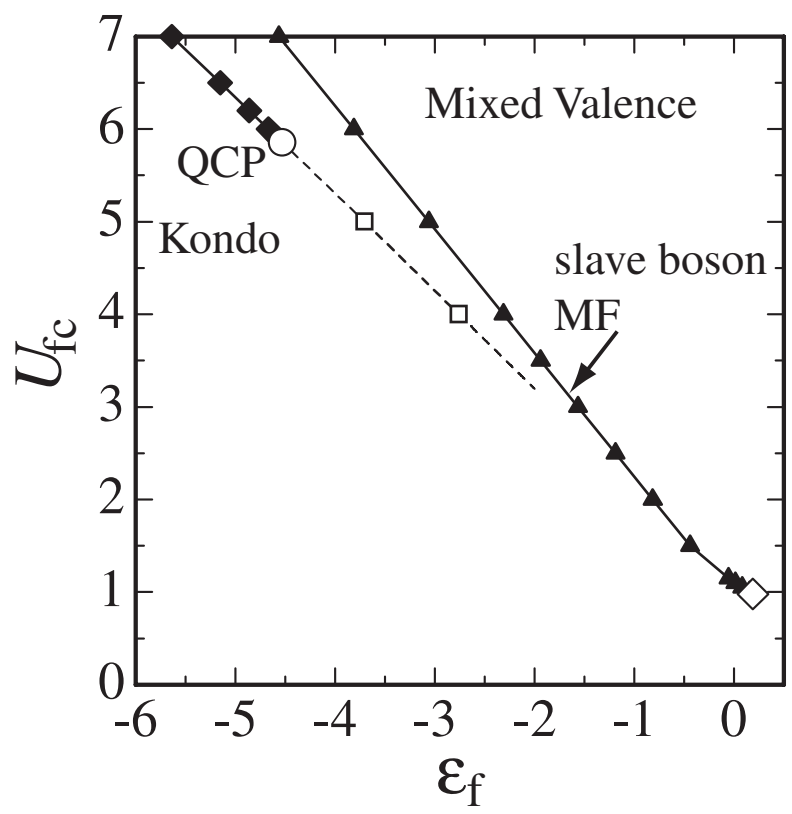

Fig. 2. Ground-state phase diagram in $U_{\mathrm{fc}}-\varepsilon_{\mathrm{f}}$ plane for $t=1, V=0.1, U=100$ at $n=7 / 8$. The firstorder valence transition line (solid line with solid diamonds) with the quantum critical point (open circle) is determined by the DMRG. The dashed line with open squares represents the crossover determined by the point where $\chi_{\mathrm{f}}$ has its maximum value. The first-order valence transition line (solid line with solid triangles) with the QCP (open diamond) is determined by the slave-boson mean-field theory.

this sharp crossover regime with strong fluctuation is most interesting, since the superconductivity is observed in this regime.

To clarify this point, we have calculated the superconducting correlation functions $\left\langle P_{m \pm}^{\mathrm{ab}}(i) P_{m \pm}^{\mathrm{cd}}{ }^{\dagger}(i+x)\right\rangle$ with $P_{m \pm}^{\mathrm{ab}}(i)=\left(a_{i \uparrow} b_{i+m \downarrow} \pm a_{i \downarrow} b_{i+m \uparrow}\right) / \sqrt{2}$ for the singlet $(-)$ and triplet $(+)$ pairing. Here, $a, b, c$ and $d$ denote the $\mathrm{f}$ and/or conduction electron operators and we have calculated the nearest-neighbor $(\mathrm{NN})$ pairing $(m=1)$ and also the onsite pairing $(m=0)$. To avoid the effect of the open boundary, we here fix the position of the operator at the central site, i.e., $i=N / 2$ for the onsite pairing and $i=N / 2-1$ for the NN pairing. Figure 3 shows the superconducting correlations for $\varepsilon_{\mathrm{f}}=-3.9$ at $U_{\mathrm{fc}}=5.0$, which is close to the sharp valence change (see Fig. 1(b)). A remarkable point here is that the power of the decay of the correlations is smaller than 2 as clearly seen, for example, in the data of $\left|\left\langle P_{0-}^{\mathrm{cf}} P_{1-}^{\mathrm{cc} \dagger}\right\rangle\right|$ and $\left|\left\langle P_{0-}^{\mathrm{cf}} P_{0-}^{\mathrm{cf}}{ }^{\dagger}\right\rangle\right|$ with dashed lines indicating $x^{-2}$. We have confirmed that all correlations of $\left|\left\langle P_{m-}^{\mathrm{ab}}(i) P_{m-}^{\mathrm{cd}}{ }^{\dagger}(i+x)\right\rangle\right|$ show nearly the same power which is smaller than 2 . On the other hand, for $\varepsilon_{\mathrm{f}}=-4.2$, which is in the Kondo regime, we find that the power of the decay of the pairing correlation is larger than 2 . It is also found that the pairing correlation decays rapidly as $\sim x^{-5}$ for $\varepsilon_{\mathrm{f}}=-3.71$, where the sharp valence crossover occurs. We confirmed that these 


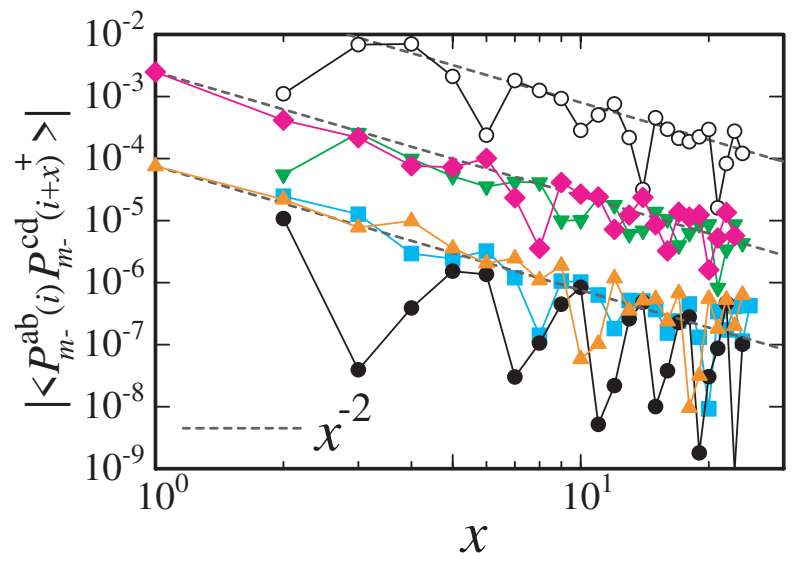

Fig. 3. Singlet pairing correlation functions $\left|\left\langle P_{m-}^{\mathrm{ab}}(i) P_{m-}^{\mathrm{cd}}{ }^{\dagger}(i+x)\right\rangle\right|$ with $P_{m-}^{\mathrm{ab}}(i)=\left(a_{i \uparrow} b_{i+m \downarrow}-\right.$ $\left.a_{i \downarrow} b_{i+m \uparrow}\right) / \sqrt{2}$ (see text) for $\varepsilon_{\mathrm{f}}=-3.9$ and $U_{\mathrm{fc}}=5.0$ at $t=1, V=0.1, U=100$ and $n=7 / 8$ : $\left|\left\langle P_{1-}^{\mathrm{cc}} P_{1-}^{\mathrm{cc}}{ }^{\dagger}\right\rangle\right|$ (black open circle), $\left|\left\langle P_{1-}^{\mathrm{ff}} P_{1-}^{\mathrm{ff}}{ }^{\dagger}\right\rangle\right|$ (black solid circle), $\left|\left\langle P_{0-}^{\mathrm{cf}} P_{1-}^{\mathrm{cc}}{ }^{\dagger}\right\rangle\right|$ (pink diamond), $\left|\left\langle P_{1-}^{\mathrm{ff}} P_{1-}^{\mathrm{cc}}{ }^{\dagger}\right\rangle\right|$ (green triangle), $\left|\left\langle P_{0-}^{\mathrm{cf}} P_{0-}^{\mathrm{cf}}{ }^{\dagger}\right\rangle\right|$ (orange triangle) and $\left|\left\langle P_{1-}^{\mathrm{ff}} P_{0-}^{\mathrm{cf}} \dagger\right\rangle\right|$ (blue square). Here, $i=N / 2$ is set for $m=0$ and $i=N / 2-1$ for $m=1$. The dashed line $\sim x^{-2}$ is drawn for comparison.

results are not changed by the calculations at several system sizes.

For $U=U_{\mathrm{fc}}=0$, the $\mathrm{f}$ level and conduction band are hybridized and $\mu$ is located at the lower hybridized band with $k_{\mathrm{F}}^{\text {large }}$. We confirmed that the charge and spin gaps are closed with $k_{\mathrm{F}}^{\text {large }}$ in the bulk limit in both the Kondo, MV and crossover regimes. Although the justification requires further careful studies, we assume the system being described by the single-band Tomonaga-Luttinger (TL) liquid in this Letter. For the single-component TL liquid without the spin and charge gaps, the singlet pairing correlation decays as $x^{-1-1 / K_{\rho}} .^{15}$ Since the charge and spin correlations decay as $x^{-1-K_{\rho}},{ }^{15}$ the above result implies that $K_{\rho}>1$, namely, that the superconducting correlation becomes dominant.

In order to estimate the TL parameter $K_{\rho}$ systematically, we made a least-squares fit for the charge, spin and superconducting correlations defined by $\left\langle\bar{O}_{i} \bar{O}_{j}^{\dagger}\right\rangle$ for $\bar{O}_{i} \equiv O_{i}-\left\langle O_{i}\right\rangle$ and $O_{i}=n_{i}^{\mathrm{c}}, S_{i}^{\mathrm{c} z}$ and $P_{m-}^{\mathrm{cc}}(i)$, respectively, since the conduction electrons have a large amplitude in the pairing correlation as seen in Fig. 3, which seems to give a reliable estimate. By assuming the asymptotic forms of the correlation functions of the single-component TL liquid, ${ }^{15}$ we obtained three $K_{\rho}$ 's from the singlet-superconducting (SS), the charge-density-wave (CDW) and the spin-density-wave (SDW) correlation functions. Then, we take the middle point between their maximum and minimum values as a mean value, with the error defined by the difference between the middle point and the maximum (minimum) value.

The $K_{\rho}$ values evaluated in this manner are shown in Fig. 1 as the solid diamonds. Here, for $\varepsilon_{\mathrm{f}} \geq-3.75$ at $U_{\mathrm{fc}}=5.0$ and for $\varepsilon_{\mathrm{f}} \geq-5.6$ at $U_{\mathrm{fc}}=7.0, K_{\rho}$ is estimated from $4 k_{\mathrm{F}} \mathrm{CDW}$ 
and SS correlations. For $U_{\mathrm{fc}}=5.0, K_{\rho}$ appears to exceed 1 at around $\varepsilon_{\mathrm{f}}=-3.9$ in Fig. $1(\mathrm{~b})$, suggesting that the superconducting correlation is dominant, which is consistent with the result presented in Fig. 3. For $U_{\mathrm{fc}}=7.0, K_{\rho}$ jumps at the FOVT as in Fig. 1(a). For $U_{\mathrm{fc}}=0$, $K_{\rho}$ estimated from the SS correlation remains smaller than 1, indicating that the pairing correlation does not develop as in Fig. 1(c). These results show that the superconducting correlation is enhanced in the Kondo regime near the sharp valence crossover, which is close to the QCP of the valence transition.

To get further insight into the nonmonotonic behavior of $K_{\rho}$ around $\varepsilon_{\mathrm{f}}=-3.9$ at $U_{\mathrm{fc}}=5.0$, we calculated the charge compressibility $\kappa \equiv \partial(2 n) / \partial \mu$, since the TL parameter is expressed by the charge velocity $v_{\mathrm{c}}$ and $\kappa$ as $K_{\rho}=\pi v_{\mathrm{c}} \kappa / 2$. In Fig. 4 , we plot the compressibility extrapolated to the $N \rightarrow \infty$ limit in the form of $\kappa=2 /\left(N \Delta_{\mathrm{c}}\right)$ with the charge gap $\Delta_{\mathrm{c}} \equiv[E(2 n N+2,0)+$ $E(2 n N-2,0)-2 E(2 n N, 0)] / 2$, where $E\left(N_{\mathrm{e}}, S\right)$ is the ground-state energy for the electron number $N_{\mathrm{e}}$ and total spin $S$. As $\varepsilon_{\mathrm{f}}$ increases, $\kappa$ increases, since total charge fluctuation becomes large. We see that $\kappa$ does not show any diverging enhancement at the sharp valence crossover, $\varepsilon_{\mathrm{f}} \sim-3.71$, in contrast to the enhanced $\chi_{\mathrm{f}}$. This is due to the cancellation of the leading terms between $\left\langle\bar{n}_{i}^{\mathrm{a}} \bar{n}_{j}^{\mathrm{a}}\right\rangle$ for a=f,c and $\left\langle\bar{n}_{i}^{\mathrm{f}} \bar{n}_{j}^{\mathrm{c}}\right\rangle$ in $\left\langle\left(\bar{n}_{i}^{\mathrm{f}}+\bar{n}_{i}^{\mathrm{c}}\right)\left(\bar{n}_{j}^{\mathrm{f}}+\bar{n}_{j}^{\mathrm{c}}\right)\right\rangle$, since the diagonal and off-diagonal charge correlations have the opposite signs for $U_{\mathrm{fc}}>0$.

A remarkable result here is that the compressibility stays constant around $\varepsilon_{\mathrm{f}}=-3.9$, where $K_{\rho}$ has a maximum. This indicates that the enhancement of $K_{\rho}$ is caused by the enhancement of the charge velocity, but not by the compressibility. This is quite different from the ordinary models, which exhibits $K_{\rho}>1 .{ }^{16}$ In order to get further insight into this finding, we calculated the charge velocity defined by $v_{\mathrm{c}}^{*} \equiv \lim _{N \rightarrow \infty} \Delta_{\mathrm{c}} / \Delta q$ with $\Delta q=\pi /(N+1)$ instead of $v_{\mathrm{c}}{ }^{17}$ since the numerical accuracy is not enough for the second-lowest eigenvalue in the Lanczos diagonalization. We see that the maximum of $v_{\mathrm{c}}^{*}$ appears around $\varepsilon_{\mathrm{f}}=-3.9$ and this suggests that the enhancement of $v_{\mathrm{c}}$ is the origin of the enhancement of $K_{\rho}$. In Fig. 4 , we show $K_{\rho}^{*}=\pi \kappa v_{\mathrm{c}}^{*} / 2$ for $\varepsilon_{\mathrm{f}} \leq-3.85$ by open diamonds and note that $K_{\rho}^{*}$ coincides with the $K_{\rho}$ obtained by the least-squares fit of the correlation functions within the error bars. Since $K_{\rho}^{*}$ is obtained by $v_{\mathrm{c}}^{*}$ and $\kappa$ after the extrapolation to $N \rightarrow \infty$, this suggests that $K_{\rho}$ is evaluated properly within the finite sizes calculated in this study. A more accurate determination of $K_{\rho}$ in the bulk limit is left for future study.

The increase in $v_{\mathrm{c}}^{*}$ toward $\varepsilon_{\mathrm{f}} \simeq-3.9$ in the Kondo regime is considered to be due to the decrease in the density of states at the Fermi level, i.e., the broadening of the Kondo peak. As $\varepsilon_{\mathrm{f}}$ further increases, $v_{\mathrm{c}}^{*}$ is suppressed, since $U_{\mathrm{fc}}$ interrupts the coherent motion of the electrons in the MV state. This is the reason why $v_{\mathrm{c}}^{*}$ has a maximum near the sharp valence increase. The enhanced $v_{\mathrm{c}}^{*}$ implies the enlargement of the effective band width and thus the coherent motion of electrons with enhanced valence fluctuation is considered to be the origin of the development of the pairing correlation. The enhancement of $K_{\rho}$ around 


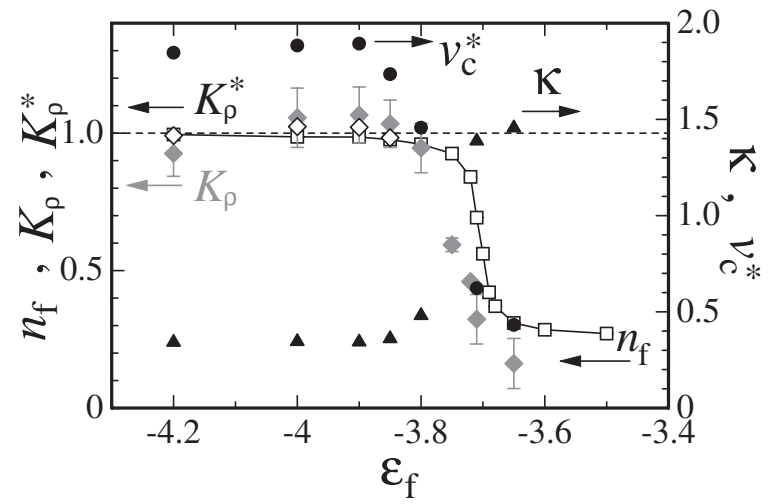

Fig. 4. Compressibility (solid triangle) obtained by $\kappa=\lim _{N \rightarrow \infty} 2 /\left(N \Delta_{\text {c }}\right)$ and charge velocity (solid circle) obtained by $v_{\mathrm{c}}^{*}=\lim _{N \rightarrow \infty} \Delta_{\mathrm{c}} / \Delta q$ for $t=1, V=0.1, U=100$ and $U_{\mathrm{fc}}=5.0$ at $n=7 / 8$. The $K_{\rho}$ (shaded diamond) obtained by the least-squares fit of correlation functions, $K_{\rho}^{*}=\pi v_{\mathrm{c}}^{*} \kappa / 2$ (open diamond), and $n_{\mathrm{f}}$ (open square) are shown.

$\varepsilon_{\mathrm{f}}=-3.9$ also agrees with the following naive expectation: In the deep Kondo regime, the SDW correlation is dominant by the RKKY interaction and at the sharp valence crossover point the CDW correlation becomes dominant with enhanced $\chi_{\mathrm{f}}$. Between the two regimes, the superconducting correlation can be dominant, since the regime midway between the Kondo state and the sharp valence crossover is unfavorable for both the SDW and CDW correlations.

To summarize, we have clarified the novel nature of the electronic states near the QCP of the valence transition. Since valence fluctuations are basically ascribed to atomic origin, we believe that the basic nature of the valence transition and effect of its fluctuation revealed in this study is universal in two- and three-dimensional systems as well.

One of the authors (S. W.) thanks H. Harima and S. Kawasaki for fruitful discussions. This work is supported in part by the Creative Research Fund (No. 15G0213) from the Japan Society for the Promotion of Science. 


\section{References}

1) D. Jaccard, et al.: Physica B 259-261 (1999) 1.

2) K. Miyake, et al.: Solid State Commun. 71 (1989) 1149.

3) T. M. Rice and K. Ueda: Phys. Rev. B 34 (1986) 6420.

4) A. T. Holmes, et al.: Phys. Rev. B 69 (2004) 024508.

5) H. Q. Yuan, et al.: Science 302 (2003) 2104.

6) S. Kawasaki, et al.: Phys. Rev. Lett. 94 (2005) 037007.

7) D. C. Koskenmaki and K. A. Gschneidner, "Handbook on the Physics and Chemistry of Rare Earths", edited by K. A. Gschneidner and L. Eyring (North-Holland, Amsterdam, 1978) Vol. 1, Chap. 4.

8) Y. Onishi and K. Miyake: J. Phys. Soc. Jpn. 69 (2000) 3955.

9) P. Monthoux and G. G. Lonzarich: Phys. Rev. B 69 (2004) 064517.

10) S. R. White: Phys. Rev. Lett. 69 (1992) 2863, S. R. White: Phys. Rev. B 48, (1993) 10345.

11) I. Singh, et al.: Solid State Commun. 34 (1980) 65.

12) Y. Onishi and K. Miyake: Physica B 281-282 (2000) 191.

13) S. Watanabe and M. Imada: J. Phys. Soc. Jpn. 73 (2004) 3341.

14) S. Watanabe: unpublished.

15) H. Schulz: Int. J. Mod. Phys. B 5 (1991) 57.

16) M. Ogata, et al.: Phys. Rev. Lett. 66 (1991) 2388.

17) When the charge velocity $v_{\mathrm{c}}$ is defined by $v_{\mathrm{c}} \equiv \lim _{\Delta q \rightarrow 0} \Delta_{\mathrm{c}}^{*} / \Delta q$ for $\Delta_{\mathrm{c}}^{*} \equiv E_{1}(2 n N, 0)-E(2 n N, 0)$ with $E_{1}$ being the first-excited state, $K_{\rho}=\pi v_{\mathrm{c}} \kappa / 2$ holds. If the symmetric condition $\varepsilon_{\mathrm{f}}+U / 2=0$ and $U_{\mathrm{fc}}=0$ are satisfied in eq. (1), $\Delta_{\mathrm{c}}=\Delta_{\mathrm{c}}^{*}$; i.e., $v_{\mathrm{c}}=v_{\mathrm{c}}^{*}$ holds by charge $\mathrm{SU}(2)$ symmetry. It is expected that $v_{\mathrm{c}} \sim v_{\mathrm{c}}^{*}$ holds for eq. (1) in the Kondo regime. 